\title{
Exploration for Skeleton CT Image Processing Technology based on RP
}

\author{
Jinxia Li \\ Xi'an Medical University, Xi'an, 710000, China
}

\begin{abstract}
CT image processing technology is an important technique which can realize rapid prototyping. According to two-dimensional information displayed in skeleton CT image, we can apply filtering processing, binarization processing and other techniques to extract necessary image edge contour, reconstruct relevant three-dimensional CAD model and transform it into the format of rapid prototyping, so as to obtain the clearest model. This technology is of very important applying value in the field of modern medicine. In this paper, the author summarizes skeleton CT image processing based on RP, and analyzes skeleton CT image processing technology based on RP.
\end{abstract}

Key words: RP, skeleton, CT image processing technology

Based on the principle of dispersion-deposition formation, rapid prototyping (RP) is a kind of new technology combining many technologies, such as computer aided technology, numerical control technology, laser technology and new material technology. Since the appearance of RP technology in the 1980s, it is gradually applied from industrial enterprise production into the art field, education field, medical science field and others. Considering good development prospect and use effect of RP technology, official governmental agencies, enterprises and research institutes in major advanced industrial countries in the world have invested los of funds into implementation, research and development of this technology. Due to proud influence on the field of medicine science, however, the key CT image processing technology in RP technology has also become a key link of research.

\section{Overview of Skeleton CT Image Processing based on RP}

To implement the skeleton RP technology under CT image, we must first process skeleton CT image, so as to obtain vector data on skeleton outline. The general image processing process is to process filtering first, implement equalization, extract edge, perform binarization, detect image edge and obtain the outline. Generally, skeleton CT image is composed of two parts: soft tissue and sclerotin. Soft tissue and skeleton have respective density. Moreover, rather big difference exists between soft tissue and skeleton. In the image, the range of gray value with relatively great difference is mainly presented. In general, skeleton gray level is between 210 and 255. However, the gray level of other tissues is between 80 and 150. Based on relevant theories of digital image processing and the skeleton brightness of CT image should exceed the brightness of other tissues, the author proposed the skeleton CT image processing method based 
on RP. In fact, the first is to realize filtering and noise reduction. The second is to implement binarization. The third is to extract contour. The fourth is to finish vectorization.

\section{Analysis on Skeleton CT Image Processing Technology based on RP (I) Skeleton CT image filtering processing technology}

CT image may produce various kinds of parasitic effects. However, these parasitic effects may appear during quantization of image collection. Moreover, these parasitic may also form due to the influence from all kinds of interference signals in the transmission process. At the same time, these parasitic effects will also influence the identification for CT image edge. On that account, CT image filtering should be implemented and parasitic effects should be eliminated in the process of image processing. Common filters mainly include low pass filter and median filter. For a comparison of different filtering effects of the two categories of filter, different masking matrixes can be used to compare the filtering of the same image. According to the comparative result, the former will make skeleton edge to become more and more blur. At the same time, scaleplate, mark number and other redundant information can not be completely removed in the image. Therefore, the filtering effect is unremarkable. However, the latter is of remarkable filtering effect. At the same time, it can also eliminate all kinds of unnecessary scaleplate and notes in the process of post processing. More noise can be eliminated by properly enlarging the filtering window.

\section{(II) Skeleton CT image binarization processing technology}

Skeleton CT image binarization is to select the most reasonable gray level threshold and then use this threshold to make a reasonable comparison to the gray level of pixel at various points in the image. Once it exceeds the threshold, a relevant gray level should be given accordingly. Once it is lower than the threshold, another new gray level should be given accordingly. There are many methods for specifying the threshold, mainly including the followings: i) grey level histogram trough method; ii) simple statistical method; iii) the minimum cross entropy method; and iv) the maximum between-cluster variance partition method. The four methods have respective characteristics and fields of application. CT image histogram is not featured by doublet. Therefore, the grey level histogram trough method fails to comply with the feature of big skeleton CT image. The single statistical method is used to analyze the mean value of gray level of the image. This mean value mainly presents the center of gray level. However, this center fails to embody the limit between skeleton and soft tissue, especially when there is great difference in distribution between skeleton and soft tissue. The minimum cross entropy method is to express the pixel probability distribution of the original image and the split image with $\mathrm{P}$ and $Q$ respectively and then obtain the optimal value. The minimum cross entropy method lets the minimum difference in amount of information between before the partition and post image. The cross entropy is used to measure the difference in amount of information between two different probability distributions. It belongs to a convex function. If the two probabilities are consistent, it equals to zero. The minimum cross entropy method can be used to partition the 
gray level distribution uniformity of the two parts. The threshold obtained through the experiment can favorably partition the skeleton CT image. The maximum between-cluster variance partition method is realized on the basis of the least square method. It mainly utilizes difference in characteristics between the target to be extracted in the image and the background gray level to select a reasonable threshold to specify whether each point in the image belongs to the target or is subordinate to the background. Thus, relevant binary image can be formed. This starting point is consistent with features of CT image. Verified by the experiment, the effect is the optimal. Therefore, the maximum between-cluster variance partition method is the most applicable to binarization of skeleton CT image based on RP. The emphasis of binarization is to select a reasonable threshold. Once the threshold is too high, considerable skeleton will be wrongly listed into soft tissue. On the contrary, considerable soft tissues may be wrongly portioned as skeleton, once the threshold is too low. In the practical application, the maximum between-cluster variance partition method can be used to obtain a preliminary estimated value and then device the final value according to experiences of the detector.

\section{(III) Image edge detection and outline simplification technology}

The contour extraction algorithm is mainly to hollow out internal points. Once there is a black point in the original image and adjacent 8 points are black as well, this point can be deleted. Under normal conditions, first-order or second derivative is used to detect its edge. The commonest edge detection methods mainly include sobel operator, Roberts gradient operator and Prewitt operator. At the same time, there are also a large number of new edge detection methods, such as the optimal edge detection method and wavelet analytical method proposed by Canny. Frequently used contour simplification methods include equidistance sampling method, curvature sampling method and chord difference method. The equidistance sampling method will lead to the disappearance of many feature points during specific implementation. However, the curvature sampling method may generate second derivative in the algorithm, so as to increase the calculated amount. Therefore, the chord difference method is commonly used. At present, the optimal detection method proposed by Canny is used at most cases at present. This method was first proposed by Canny in 1986. It is an edge detection method with favorable signal to noise ratio and detection precision. It has been widely applied into image processing. This method is the best for phase step edge under the influence from white noise. Main standards for the optimality are listed as follows: the first one is detection standard. Signal to noise ratio with good quality will not loose important edges or generate false edges. The second one is positioning standard. The deviation of position between substantial edge and detected edge is the minimum. The third one is response standard. Single edges must have only response, so as to control the response to false edges to the maximum extent. After convolution of the image and the symmetry gaussian function, differential is performed along the gradient direction, so as to form single and effective operator. 


\section{(IV) Outline vectorization and inner \& outer boundary identification technology}

So-called contour vectorization is mainly to utilize mathematical function to define this category of vector curves, so as to specify the contour of an object. It is necessary to restrict points in both the curve and the contour into a certain tolerance in the perpendicular distance. The generally used method is to select the peak of the initial segment in the contour, sequentially select other sectional points according to some principles, use a certain vector curve to interpolate into sectional points, and then control the tolerance into relevant threshold. Thus, vector lines of the contour are formed. The emphasis of vectorization is to identify and select sectional points. At present, the solution to this issue is divided into two categories: the first one is to directly determine through the boundary contour; and the second one is to be approximate to detection with piecewise linearity restrained by a close degree. For instance, tolerance is used in the polygonal approximation algorithm to judge the colinearity of points in a certain section of the edge and a straight line. Once the distance from points in a certain section to the straight line is within the tolerance, this section of edge can be approximate to with a straight line. Or, the point with the longest distance from the straight line will become a new segment point. While implementing three-dimensional reconstruction and forming STL document of model, it needs to judge whether the reconstruction surface belongs to the internal surface or the external surface and whether the triangular patch is on the internal surface or the external surface. The triangular peaks of the internal surface or the external surface are difference in terms of reasonable marshalling sequence. Therefore, internal and external line properties of the contour should be judged first.

After contour extraction and vectorization, cross-sectional images will usually appear several different contour lines. According to the author, any point can be selected from a certain contour. If this point is in another contour, the principle of non-crossing of two arbitrary contours should be used, letting a certain contour to be effectively included into another contour. Main solutions to this issue include corner method, mark number method, cross product judgment method and included angle sum detection method. To avoid many contours included in each section, a large number of sections, great calculated amount and low efficiency in specific application, the counting of point of intersection is always used to judge the inclusion relation between point and area. The point of intersection between this horizontal radial and other contours can be judged by arbitrarily select a point in candidate contour lines and then making a radial in the horizontal or vertical direction. The number of points of intersection between the radial and the contour can be obtained. The intersection can be divided into three conditions: i) once the number of points of intersection between the radial and the contour line is an odd number, then this point is within the contour line. This contour line is one within the contour line crossed with it. Once the number of points of intersection with other contour lines is an even number, then this point is out of other contour lines. This contour line is an external one; ii) once the radial passes through the peak of the contour line, it must be especially treated. Hence, it should be 
prescribed that the number of points of intersection is 2, suppose both ends of the sharing peak are at the same side of the radial. Or, the number should plus 1 ; and iii) if there is no point of intersection between the radial and other contour lines, then this point must be out of other contour lines. The contour line with the point belongs to an external one.

The obtained sectional image is subject to alignment up and down. Moreover, contour faces in the middle are supplemented to obtain a wireframe model with vertical contour lines and closed cross section contour lines. Then, the formed triangular patch surface model is transformed into document in the format of STL capable of RP. This kind of document is actually a kind of model document obtained from three-dimensional entity model after triangle processing. It is mainly of a kind of port protocol set by American 3D SYSTEM Company. It is deemed as the current standard in the circle. This is a new data model using small planes of considerable spatial triangles to be approximate to the original CAD entity. In the process of dispersing the surface of CAD into triangular facets in different precisions, triangular net with difference is used to division. Each triangular facet in STL document is set into four different data items, namely coordinates of the three peaks and outer normal vector of triangular facets.

\section{Conclusion}

To sum up, the author in this paper made a research on skeleton image, unscrambled the skeleton CT image processing technology and specified the most reasonable processing approaches in various links. In the skeleton CT image processing technology, the median filter method should be used to implement comprehensive pre-processing and obtain the overall quality of the image through filtering all kinds of noise in the image. On this basis, CT image should be subject to binarization processing. The most ideal method should be selected by a comparison of binarization approaches, so as to successfully separate necessary information from CT image for the research on skeleton. Then, main contour of the skeleton should be extracted. On this basis, the mathematical morphology is used for detailed partition of contour, so as to obtain specific coordinates of all points in the contour. Finally, relevant contour data tracked should be subject to vectorization processing, so as to realize vectorization of the contour and identification for internal \& external edges. Fact has proved that the skeleton contour obtained through above methods is clear and complete. Moreover, it is also of great reference value for both skeleton CAD/CAM and RP system.

\section{References:}

[1] Xiao Riguo, Discussion on Three-dimensional Reconstruction of Skeleton CT Image and Its Clinical Significance [J], Henan Journal of Surgery, 2009 (6);

[2] Li Xiaolin, Application of Mimics Software in Fast Construction of Human Body Thoracolumbar Skeleton Finite Element Model based on CT Image [J], Journal of Clinical Rehabilitative Tissue Engineering Research, 2009 (39);

[3] Qian Zhihui, Reconstruction of Three-dimensional Entity Model of Human Body Lower Limb Skeleton based on CT Image [J], Journal of Jilin University (Engineering and Technology 
Edition), 2012 (1);

[4] Huang Jiale, Application of Spiral CT Image Post-processing in Arthrotrauma Examination [J], Contemporary Medicine, 2013 (8);

[5] Zhao Guofeng, Discussion on Diagnostic Value of Multi-level CT Image Post-processing Technology for Incomplete Rib Fracture [J], Chinese Community Doctors, 2014 (2) 